AperTO - Archivio Istituzionale Open Access dell'Università di Torino

\title{
Electrocatalysis in the oxidation of acetaminophen with an electrochemically activated glassy
} carbon electrode

\section{This is the author's manuscript}

Original Citation:

Availability:

This version is available http://hdl.handle.net/2318/1558784

since 2019-09-11T14:44:39Z

Published version:

DOI:10.1016/j.electacta.2016.01.187

Terms of use:

Open Access

Anyone can freely access the full text of works made available as "Open Access". Works made available under a Creative Commons license can be used according to the terms and conditions of said license. Use of all other works requires consent of the right holder (author or publisher) if not exempted from copyright protection by the applicable law. 


\section{(5) \\ UNIVERSITÀ DEGLI STUDI DI TORINO}

This Accepted Author Manuscript (AAM) is copyrighted and published by Elsevier. It is posted here by agreement between Elsevier and the University of Turin. Changes resulting from the publishing process - such as editing, corrections, structural formatting, and other quality control mechanisms - may not be reflected in this version of the text. The definitive version of the text was subsequently published in Electrochimica Acta, 192, 29 January 2016, 10.1016/j.electacta.2016.01.187.

You may download, copy and otherwise use the AAM for non-commercial purposes provided that your license is limited by the following restrictions:

(1) You may use this AAM for non-commercial purposes only under the terms of the CC-BY-NC-ND license.

(2) The integrity of the work and identification of the author, copyright owner, and publisher must be preserved in any copy.

(3) You must attribute this AAM in the following format: Creative Commons BY-NC-ND license (http://creativecommons.org/licenses/by-nc-nd/4.0/deed.en),

http://dx.doi.org/10.1016/j.electacta.2016.01.187 
Electrocatalysis in the oxidation of acetaminophen with an electrochemically activated glassy carbon electrode

Enrico Chiavazza ${ }^{1^{*}}$, Silvia Berto ${ }^{1}$, Agnese Giacomino ${ }^{2}$, Mery Malandrino ${ }^{1}$, Claudia Barolo $^{1,3}$, Enrico Prenesti ${ }^{1}$, Davide Vione ${ }^{1}$, Ornella Abollino ${ }^{1}$

${ }^{1}$ Dept. of Chemistry, University of Torino, via P. Giuria 7, 10125 Turin, Italy

${ }^{2}$ Dept. of Drug Science and Technology, University of Torino, Via Giuria 9, 10125 Turin, Italy

${ }^{3}$ INSTM and NIS Centre, University of Torino, Via Quarello 15A, 10135 Turin, Italy

\begin{abstract}
A simple approach such as the anodic activation of a glassy carbon electrode (GCE) can be used successfully to improve the detection of acetaminophen at very low concentration levels. In this work, we observed that the exposure of a GCE to a high potential ( $2 \vee v s$ $\mathrm{Ag} / \mathrm{AgCl}$ ) for a limited time period $(60 \mathrm{~s})$ in the presence of $50 \mathrm{mM}$ borate/phosphate buffer ( $\mathrm{pH}$ 9) provides a strongly electro-activated surface. The activated surface was characterized by means of several techniques (electrochemistry, EIS, SEM, AFM, $\mu-$ Raman, XPS). It appeared that the anodization procedure gave rise to a strong oxygenbased functionalization that did not affect morphologically the electrode surface. The mechanism of interaction of the activated electrode with the analyte was studied by electrochemical methods, then the electrode was applied to the electroanalysis of acetaminophen by differential pulse voltammetry. In this application, the method showed promising analytical performance, detecting nanomolar traces of acetaminophen.
\end{abstract}

\title{
Keywords
}

Acetominophen oxidation; Glassy carbon electrode; Anodization; Electrochemical activation; 


\section{Introduction}

The occurrence of pharmaceuticals in the environment and, in particular, in surface waters is a problem of increasing concern [1]. Excretion by humans of non- or partially metabolized molecules, incorrect disposal of expired medicines and use of antibiotics for cattle breeding are the main routes of pharmaceuticals to the environment. The compounds identified in various environmental compartments belong to several classes of human drugs including analgesics, antibiotics, beta-blockers, anticonvulsants, lipid regulators, contrast agents, anti-cancer agents and hormones [2]. They may be toxic to fish and aquatic invertebrates [3], have endocrine disruption effects [4] and favor the occurrence of resistance in bacteria [5]. The purification processes of wastewaters, such as those based on activated sludge, are often unable to remove these pollutants efficiently, because wastewater treatment plants (WWTPs) were not originally designed to eliminate xenobiotics. Analgesics, anti-inflammatories and beta-blockers are among the most resistant pharmaceuticals to the WWTP treatment (30-40\% of removal efficiency), while the removal of antibiotics can reach $50 \%[6]$.

The monitoring of the environmental contamination by pharmaceuticals is worthwhile for several reasons, which include the reliable assessment of risks for the ecosystem and, through the food chain, for humans, as well as the evaluation and maintenance of the water resource quality.

Several research papers report on the application of electrochemical methods for the quantification of drugs, and in the last ten years the use of carbon electrodes in this field has considerably increased [7]. Acetaminophen (AP) is one of the most extensively employed painkiller drugs in the world and, for this reason, its occurrence in the environment is reported at concentrations of up to $10 \mu \mathrm{g} / \mathrm{L}$ [8]. Several electrochemical methodologies have been used for AP determination: most of them involve hybrid or 
composite glassy carbon electrodes (GCE) functionalized with gold nanoparticles, multiwalled carbon nanotubes, fullerenes, boron-doped diamonds etc. [9-13]. Recently, Sadikoglu and coworkers [14] presented a simple approach for an easy determination of AP with a bare GCE, claiming submicromolar detection. In this work, we studied and optimized a method for the AP determination at lower concentration using an electrochemically treated GCE, exploiting an anodization procedure. We report here in detail the study of the electrode surface activation process and the electrocatalytic oxidation mechanism by electrochemistry, electrochemical impedance spectroscopy (EIS), Raman spectroscopy, electron microscopy, and X-ray photoelectron spectroscopy (XPS).

\section{Experimental}

\subsection{Reagents}

Chemicals were purchased from Sigma Aldrich. Ferrocene and $\left(\mathrm{C}_{4} \mathrm{H}_{9}\right)_{4} \mathrm{NPF}_{6}$ were used, without further treatment, for the electrode area measurement, and 1,1'ferrocenedimethanol was used as probe for electrochemical characterization. Acetonitrile was distilled over $\mathrm{CaCl}_{2}$ under $\mathrm{N}_{2}$ atmosphere prior to the measurements. Deionized water $(0.1 \mathrm{M} \Omega \mathrm{cm})$ from a Millipore-RIOs 3 system was used to prepare all the solutions. The stock solution of acetaminophen (AP) was prepared in deionized water every day. The borate-phosphate buffer (hereinafter, BPB solution) was prepared by dissolving $\mathrm{K}_{2} \mathrm{HPO}_{4}$ and $\mathrm{Na}_{2} \mathrm{~B}_{4} \mathrm{O}_{7}$ salts to a final concentration of 4.5 and $0.5 \cdot 10^{-2} \mathrm{M}$, respectively, and adjusting the $\mathrm{pH}$ to $9.0 \pm 0.1$ with $\mathrm{H}_{3} \mathrm{PO}_{4} 80 \%$. 


\subsection{Instrumentation}

The electrochemical measurements and treatments were performed with a Bio-Logic SP150 potentiostat. A conventional three-electrode system in a thermostated cell (NESLAB RTE-101 thermostat) was used. Electrochemically activated GCE $\left(3 \cdot 10^{-3} \mathrm{~m}\right.$ diameter, from ALS) was used as working electrode; a platinum wire was used as counter and $\mathrm{Ag} / \mathrm{AgCl}, 3 \mathrm{M} \mathrm{KCl}$ (Metrohm, Model 6.0733.100) as reference electrodes, except where otherwise stated. A magnetic stirrer provided the convective transport during the amperometric and differential pulse voltammetric measurements. Electrochemical Impedance Spectroscopy (EIS) experiments were performed using the built-in frequency response analyzer fuction of the same potentiostat.

Rotation Disk Electrode linear scan voltammetry (RDE-LSV) experiments were performed on a Eco Chemie Autolab PGSTAT 10 potentiostat with a 663 VA Stand, GCE $\left(2 \cdot 10^{-3} \mathrm{~m}\right.$ diameter, Metrohom) was used as working electrode; a GCE rod (Metrohm) was used as counter and $\mathrm{Ag} / \mathrm{AgCl}, 3 \mathrm{M} \mathrm{KCl}$ (Metrohm) as reference electrodes. The potential range employed was between 0.1 and $0.8 \mathrm{~V}$, the working electrode was rotated at rates ranging between 500 and 3000 rpm.

The $\mathrm{pH}$ was measured with a combined glass-membrane electrode controlled by a XS pH6 pH-meter (CRISON).

Scanning electron microscope (SEM) images were acquired with a Quanta ${ }^{\mathrm{TM}}$ 3D FEG DualBeam $^{\mathrm{TM}}$ electron microscope, and Raman spectra were obtained with a Thermo Scientific DXR Raman microscope. The XPS spectra were taken with a PHI 5000 VERSA PROBE, Physical Electronics X-ray photoelectron spectrometer, equipped with a monochromatic Al source and a hemispherical analyzer on a glassy carbon plate $(25 \times 25 \times 3$ $\mathrm{mm}$, Alfa-Aesar). Survey scans as well as narrow scans (high resolution spectra) were 
recorded with a $1 \cdot 10^{-4} \mathrm{~m}$ spot size on both, pristine and activated regions. Data acquisition and processing were implemented with the instrument software Summit and MultiPak 8.2. For internal calibration, the XPS spectrometer was shifted so that the Carbon peak lay at $284.80 \mathrm{eV}$. The morphology of the films was studied by Atomic Force Microscopy (AFM) by means of a Park System XE-100 microscope, with a silicon tip (Park System) operating in non-contact mode.

\subsection{Electrochemical procedures}

The electrochemical procedures were optimized as detailed in the Results and Discussion paragraph. The conditions thus optimized and used for the AP quantification are summarized below.

\subsubsection{Activation step}

The working electrode was mechanically polished with diamond powder ( $1 \mu \mathrm{m}, \mathrm{ALS})$ and alumina $(0.3 \mu \mathrm{m}, \mathrm{ALS})$, thoroughly washed with ethanol and deionized water, placed in the BPB solution and activated by applying an anodic potential of $2.0 \mathrm{~V}$ for $60 \mathrm{~s}$ under magnetic stirring (500 rpm). The activation was followed by chronoamperometry. The activated electrode was then directly used for the measurements.

\subsubsection{Measurements}

The electrochemical experiments were carried out in the sample solution spiked with an aliquot of borate-phosphate buffer in order to obtain a $5 \cdot 10^{-2} \mathrm{M}$ final concentration $(\mathrm{pH}$ 9.0). Each measurement was composed of a brief conditioning period ( $5 \mathrm{~s}$ ) at $2.0 \mathrm{~V}$. The parameters were the following: step height $5 \cdot 10^{-3} \mathrm{~V}$, pulse amplitude $5 \cdot 10^{-2} \mathrm{~V}$, pulse length $5 \cdot 10^{-2} \mathrm{~s}$, pulse period $0.100 \mathrm{~s}$ and a potential range between $0 \mathrm{~V}$ and $0.400 \mathrm{~V}$. All the experiments were conducted at $25.0 \pm 0.5^{\circ} \mathrm{C}$. Two operational modalities were highlighted: 
the highest sensitivities could be reached by repeating 10 times the DPV sequence after the initial conditioning (the beneficial effect has been observed using the reported EC-Lab instrument and it has not been assessed on other potentiostats); in the second modality only a DPV scan was executed, which increased the limit of detection by one order of magnitude, although it was more practical. All the measurements in this paper followed the first modality.

\section{Results and Discussion}

\subsection{Method Optimization}

The variables involved in our procedure could be divided into those regarding the activation step and others related to the DPV measurement. Activation time, potential and electrolyte were optimized independently. The best conditions were found using the mixed phosphate-borate buffer at $\mathrm{pH} 9$, with an anodizing time of $60 \mathrm{~s}$ at a potential of $2.0 \mathrm{~V} v \mathrm{~s}$. $\mathrm{Ag} / \mathrm{AgCl}, 3 \mathrm{M} \mathrm{KCl}$. The evaluation was based on the repeatability of the measurements and on the intensity of the DPV signal. Potential values lower than $1.5 \mathrm{~V}$ did not increase the sensitivity toward the analyte, while potentials higher than $2.0 \mathrm{~V}$ caused strong gas evolution. Moreover, anodization periods longer than $1 \mathrm{~min}$ did not increase significantly the current (Supplementary Material, Figure S1a). A second brief conditioning period during the measurement step, even in the presence of the analyte, proved useful to maintain a stable activation. One activation could last several complete measurements without a reduction of the DPV signal. However, when the measurements are distribuited across more days it is advisable to renew the electrode each time it has to be used, since the renewal of the surface is easy and the electrochemical activation very reproducible (see paragraph 3.5). The activation procedure allowed an increase of sensitivity of 2 order 
of magnitude, when compared to a traditional AP determination using a non activated GCE (LoD 3.69-10 $\left.10^{-7} \mathrm{M}[14]\right)$.

The chronoamperometric (CA) response of the electrode during the activation process showed a characteristic current increase a few seconds after the zeroing of the capacitive current contribution (Fig. 1a). Interestingly, the CA response and the consequent electroanalytical performance appeared to be strongly dependent on the choice of the anodizing electrolyte: by comparing the CA and the DPV curves for different electrolytes at the same $\mathrm{pH}$ value, high currents for the AP measurement were observed only when the phosphate or borate anions (see Supplementary Material, Figures S2) were used as anodizing electrolyte. The cyclic voltammetry after the anodization in the presence of BPB as electrolyte, showed a huge increase of the cathodic current at very low potential $(-1.8$ and $-2.1 \mathrm{~V}$ vs $\mathrm{Ag} / \mathrm{AgCl}, 3 \mathrm{M} \mathrm{KCl}$ ). Much lower intensity was observed when $0.1 \mathrm{M} \mathrm{KCl}$ at $\mathrm{pH} 9$ was used as anodizing electrolyte (Fig. 1c).

The best $\mathrm{pH}$ was found to be 9 because of the lower background found from 0 to $0.5 \mathrm{~V}$. Moreover, as reported in the literature [9], at lower $\mathrm{pH}$ the $\mathrm{E}_{1 / 2}$ of $\mathrm{AP}$ is shifted to higher values because of the proton role in the homogenous hydrolysis reaction (Fig. 2). The best performing electrolyte was sodium phosphate, but the phosphate buffer shows a minimal buffer capacity at $\mathrm{pH}$ 9. Therefore, a small quantity of borate buffer was added to increase the buffer capacity, without strongly affecting the sensitivity of the electrode. A drift of the AP redox potential as a function of $\mathrm{pH}$ is observed also with the activated electrode $\left(5.5 \cdot 10^{-2} \mathrm{~V} / \mathrm{pH}\right)$ and, for that reason, a proper buffer is necessary.

The optimized parameters characterizing the DPV run were the following: pulse amplitude $\left(5 \cdot 10^{-2} \mathrm{~V}\right)$, pulse length $\left(5 \cdot 10^{-2} \mathrm{~s}\right)$, step height $\left(5 \cdot 10^{-3} \mathrm{~V}\right)$ and repetition period $(0.100 \mathrm{~s})$ (Supplementary Material, Figure S3). Such a combination was not the one with the highest 
absolute DPV signal, but it was a compromise between intensity and sharpness of the peak.

\subsection{Characterization of activated GCE}

The effect of an anodic overpotential has been often explained as an extensive oxidation of the graphite surface of the electrode $[15,16]$. As result of that, the introduction of polar oxygenated functions (hydroxyl, carbonyl, carboxyl and oxide groups) has been reported when GCEs were anodized in strongly basic media (1 $\mathrm{M} \mathrm{NaOH})$ [17]. However, if such harsh conditions were maintained for relatively long periods (60-120 s), the result was the detachment of a thick oxide layer (up to $1 \cdot 10^{-6} \mathrm{~m}$ ) and the substantial damaging of the mirror-like surface.

Although in our activation process we exposed the graphitic surface to anodic conditions, the surface anodization in bland basic media $(\mathrm{pH} 9)$ maintained a mirror-finished electrode surface without evident morphologic damage to the naked eye. The chronoamperometric (CA) response of the electrode showed, as reported above, a characteristic current increase a few seconds after the zeroing of the capacitive current contribution. A plateau was then reached and the CA current was stable even after several measurements (Fig 1a). This behavior seems to suggest the onset of a chemical reaction that allows the formation of a certain number of polar groups on the electrode surface, which increases its electrical conductivity. To sustain this hypothesis, electrochemical impedence spectroscopy (EIS) was performed in a solution containing AP $5 \cdot 10^{-4} \mathrm{M}\left(\mathrm{BPB} 5 \cdot 10^{-2} \mathrm{M} \mathrm{pH}\right.$ 9, EIS from $1 \cdot 10^{5}$ to $2 \cdot 10^{-2} \mathrm{~Hz}$, sinusoidal amplitude $7 \cdot 10^{-3} \mathrm{~V}$ RMS) before and after the activation of the electrode at $\mathrm{DC}$ potentials of 0.5 and $0.2 \mathrm{~V}$, respectively. In Fig. $1 \mathrm{~b}$ the Bode plot is shown, and after the activation the charge transfer resistance $\left(R_{C T}\right)$ decreased 
from 2.1 $\mathrm{M} \Omega$ to $417 \mathrm{~K} \Omega$ at $2 \mathrm{mHz}$. The Nyquist plot is shown in the Supplementary Material file, Figure S4.

The effect of the activation was then assessed with the electrochemical probe 1,1'ferrocenedimethanol. Surprisingly it was observed a reduction of the anodic current, while the cathodic one was only shifted to a slightly lower potential $\left(E_{1 / 2}\right.$ passed from $278 \mathrm{mV}$ to $249 \mathrm{mV}$, Fig. 1d). The measured area of the electrode upon activation appeared to be almost unvaried, passing from 5.5 to $5.1 \cdot 10^{-6} \mathrm{~m}^{2}$ (Supplementary Material, Figures S5-6).

In order to assess the nature of such electrode activation, micro-Raman and XPS measurements were performed on the material prior and after the activation. In the Raman spectra (Fig. 3), the bands at $\sim 1330$ and $\sim 1600 \mathrm{~cm}^{-1}$ correspond to the graphite/graphene characteristic D and G Raman modes, respectively; in fact, the Stokes phonon energy shift caused by laser excitation creates two main peaks: $G\left(1580 \mathrm{~cm}^{-1}\right)$, a primary in-plane vibrational mode, and 2D (2690 $\left.\mathrm{cm}^{-1}\right)$, a second-order overtone of a different in-plane vibration, $D\left(1350 \mathrm{~cm}^{-1}\right)$. The first-order $\mathrm{D}$ peak itself is not visible in graphene because of crystal symmetries. As the amount of disorder increases, the Raman intensity increases for the disorder peaks $D\left(1350 \mathrm{~cm}^{-1}\right)$. The decrease in the $D / G$ band ratio from 1.63 to 1.37, revealed upon activation, suggest a reduction in the defectivity degree of the surface $[18,19]$. XPS spectra (Fig. 4a, 4b) showed a massive increase of oxygen bound to the electrode surface after the activation, with a relative abundance increasing from 6 to $30 \%$ (Tab. 1). The deconvolution of the XPS spectrum shows that the oxygenated functions are mainly phenolic and carbonyl groups (Fig. 4c-f). Two peaks at higher binding energies (293 and $295 \mathrm{eV}$ ) appear related to potassium present as counterion of the superficial carboxylate groups. SEM images up to $240000 X$ did not show any morphological change upon electrode activation (Fig. 5a). Analogously, also the analysis of the AFM images (Supplementary Material, Figure S7) showed only a slightly lower roughness of the 
electrode surface after activation. In fact, upon activation, the Z-axis displacement deviation ( $3 \sigma$ of the fitted Gaussian distribution, accounting for $98 \%$ of the data population) decreased from 9.4 to $7.8 \cdot 10^{-9} \mathrm{~m}$ (Fig. $5 \mathrm{~b}$ ). The anodization of the GCE through the analysis of the $\mu$-Raman, XPS, SEM and AFM features could be interpreted as the building of a homogeneus oxide-like layer [15].

\subsection{Reaction Kinetics}

The relationship between the peak current intensity and scan rates provides insight into the electrode redox kinetics. Figure 6 b-c-d shows the $\log (i) v s \log (v)$ plot for the electrode where $i$ is the peak current and $v$ is the scan rate. The current depends on the scan rate as $i \approx v^{\mathrm{p}}$. The coefficient $\mathrm{p}$ is derived from the Randles-Sevcik equation (see Supplementary Material, paragraph 5) and it is indicative of the phenomenon that control the kinetics in the electrode $[20,21]$. The GCE anodic current shows a linear dependence $(0.39<p<0.50)$ approximable to a common diffusion-controlled response before and after the activation[14, 22].

Experiments with a rotating disk electrode (RDE) were performed in order to get insight into both the number of electrons involved in the oxidation reaction and the nature of the AP-electrode surface interaction. The RDE is a hydrodynamic electrode technique that exploits convection as mass transport, differently from CV that is governed by diffusion. The Levich equation (Eq. 2) establishes the relationship between the limiting current at the RDE and the concentration of the analyte.

$\mathrm{i}_{\lim }=(0.620) n F A D^{2 / 3} \omega^{1 / 2} v^{-1 / 6} \mathrm{C}$

where $i_{\text {lim }}$ is the limiting current, $n$ number of electrons for the reaction, $F$ is the Faraday constant $\left(96,485 \mathrm{C} \mathrm{mol}^{-1}\right), D$ is the diffusion coefficient of AP in the solution $\left(6.64 \cdot 10^{-6} \mathrm{~cm}^{2}\right.$ $\mathrm{s}^{-1}$ [23]), $v$ is the kinematic viscosity of the solution (approximated to that of pure water at 
$0.01 \mathrm{~cm}^{2} \mathrm{~s}^{-1}$ ), C is the concentration of AP (in mol cm${ }^{-3}$ ), $\omega$ is the angular frequency ( $\mathrm{rpm}=$ $2 \pi f / 60$ ) and $A$ is the area of the electrode (in $\mathrm{cm}^{2}$ ). The RDE curves give insight into the number of electrons transferred in the electrochemical reaction by comparing the limiting currents to the square root of the rotation rate of the electrode. The Levich plots $\left(\omega^{1 / 2} v s\right.$ $\mathrm{J} / \mathrm{C}_{0}$, where $\mathrm{J}$ is the current density, defined as the current/area ratio of the electrode, and $\mathrm{C}_{0}$ the bulk concentration of AP) display well defined linear trends when the mass transfer is controlled by only one process to the electrode $[24,25]$. Interestingly, the Levich study of the behaviour of the activated GCE (Fig. 7) showed a marked peak not observed before the activation. The peak was quenched by exposing the activated electrode to a small amount of a cationic surfactant $(0.5 \mathrm{mM} \mathrm{N}$-cetyl (N,N,N-trimethyl)ammonium bromide, CTAB, Figure 7c). Moreover, the non-linearity of the Levich plot (Figure $7 \mathrm{~d}$ ) indicates that a step preceding the electro-oxidation limits the current to values below those corresponding to the convection-limited reduction of AP [24]. The slope of the experimental series regression line relate to the number of exchanged electrons, in our case this is closer to one at higher rotation rate. These outcomes can be related to a transient adsorption mechanism $[26,27,28]$ of AP on the activated surface of the electrode that appears to be responsible for the sensitization effect. The anodic activation on the electrochemistry of AP appears then to be a remarkable electrocatalysis example. In fact, upon activation the $E_{a}$ of the irreversible anodic wave shifts from $0.452 \mathrm{~V}$ to a quasi-reversible $E_{\mathrm{a}}$ of $0.301 \mathrm{mV}$, gaining a better reversibility of the electrochemical oxidation ( $\Delta \mathrm{E}$ passed from 0.522 to 0.155 V, Fig. 6b).

\subsection{Mechanism of surface interaction of $A P$}

Several molecules (Fig. 8) were tested as reactivity probes for the activated GCE. These molecules have similar structure as AP but have at least one different functional group. In 12 
this way, the effect of the electrode activation on the electrochemical response (CV) to the probe can be interpreted as the molecular moiety responsible for the interaction. The survey highlighted that only the phenol-containing molecules showed a $E_{1 / 2}$ potential downshift and a remarkable $\Delta E$ narrowing when the activated electrode was used (Supplementary Material, paragraph 7) .This means that the direct mediation of the phenolic moiety is the likely cause of the enhancement in the electrochemical response also in the case of AP. The possibility of expoiting this selectivity towards phenolic ring containing molecules could be an actractive feature for analytical applications, not developed yet in this work.

\subsection{Preliminary assessment of the analytical performance}

Fig. 9a shows the DPV curves from which the calibration curve (peak area, A, vs. AP concentration) was obtained (Fig. 9b). Each AP standard solution was analyzed 3 times with the exception of the lowest concentration, for which five repetitions were carried out to evaluate the limits of detection and quantification (LoD/LoQ). The relative standard deviation for the measurement of $1.5 \cdot 10^{-8} \mathrm{M}$ AP was $6 \%$, obtained by repeating 5 independent measurements at that concentration. From this value, by calculating the corresponding $3 \sigma$ and $10 \sigma$, indicative values of $L O D$ and $L O Q$ of $2 \cdot 10^{-9}$ and $9 \cdot 10^{-9} \mathrm{M}$ were obtained. These low values are supported by the experimental signals recorded with AP solutions in the low concentration range (see inset, Fig. 9a). The interval of linearity ranged between $2 \cdot 10^{-9}$ and $1.5 \cdot 10^{-7} \mathrm{M}$ and the straight-line had a slope of $0.00878 \pm 10^{-3} \mathrm{~A} \mathrm{M}^{-1}$. Thus, this method could be capable of detecting AP at concentration levels known to be present in water samples. Actually, the concentration of AP in surface waters often ranges between $5 \cdot 10^{-9}$ and $5 \cdot 10^{-8} \mathrm{M}[8]$. 


\section{Conclusions}

A promising application field of electro-analysis is the development of cheap and versatile trace analytical methods to be used in the study of emerging pollutants. Even a simple approach such as the anodic electrode activation can be used with success to improve the detection of organic compounds at very low concentration levels. In our work, we observed that a strong electro-active layer was formed by exposing a GCE to a high potential (2V vs $\mathrm{Ag} / \mathrm{AgCl}$ ) for a limited time period (60 s), in the presence of $5 \cdot 10^{-2} \mathrm{M}$ borate/phosphate buffer ( $\mathrm{pH}$ 9). This activated layer was characterized with SEM, Raman, XPS, AFM, EIS and RDE in order to understand its nature. SEM and AFM images showed a homogenous and perfectly adherent layer and the impedance measurements showed that the activated electrode surface is four time less resistive than the original surface. From the chemical point of view, such layer appears as an ordered and compact oxide structure and has a specific electrochemical response to the phenol-containing molecules. The layer formation strongly depends on the presence of a weak oxygenated acidic anion as electrolyte (phosphate, borate) with poorly delocalized charge. The activated electrode was used in the electro-analysis of acetaminophen by DPV. In this application, the activation is a helpful tool in improving the analytical performance of the electrode, yielding nanomolar detection capability $\left(\mathrm{LoD}=2 \cdot 10^{-9} \mathrm{M}\right.$ in borate/phosphate buffer $(\mathrm{pH} 9)$ ), with a linear response up to $1.5 \cdot 10^{-7} \mathrm{M}$. 


\section{Acknowledgments}

MM and SB acknowledge financial support from Compagnia di San Paolo, Torino, Italy (Project "Development of an Optical Sensor for the Determination of Pharmaceutical Compounds and of Their Photoreactivity in Aqueous Solution - PHARMASENS"). The same project also provided financial support for the post-doc fellowship of EC. The authors acknowledge Dr. E. Enrico for the acquisition of SEM images at NanoFacility Piemonte, INRiM, a laboratory supported by Compagnia di San Paolo; Dr. A. Giovannozzi and Dr. A. Rossi at INRiM for the $\mu$-Raman measurements and Dr. E. De Laurentiis, Dr. F. Sordello and Prof. C. Minero at Dept. of Chemistry, Univ. of Torino for the TOC measurements on water samples and the AFM analysis, finally Ing. Sara Ferraris for her collaboration in the XPS data interpretation. 


\section{References}

1. R. P. Schwarzenbach, B. I. Escher, K. Fenner, T. B. Hofstetter, C. A. Johnson, U. von Gunten, B. Wehrli, The Challenge of Micropollutants in Aquatic Systems, Science 313 (2006) 1072. DOI:10.1126/science.1127291

2. B. Halling-Sorensen, S. N. Nielsen, P. F. Lanzky, F. Ingerslev, H. C. Lutzhoft, S. E. Jorgensen, Occurrence, fate and effects of pharmaceutical substances in the environment--a review, Chemosphere 36 (1998) 357. DOI:10.1016/S0045$6535(97) 00354-8$

3. S. Kar, K. Roy, First report on interspecies quantitative correlation of ecotoxicity of pharmaceuticals, Chemosphere 81 (2010) 738. DOI:10.1016/j.chemosphere.2010.07.019

4. K. A. Kidd, P. J. Blanchfield, K. H. Mills, V. P. Palace, R. E. Evans, J. M. Lazorchak, R. W. Flick, Collapse of a fish population after exposure to a synthetic estrogen, Proc. Natl. Acad. Sci. U.S.A. 104 (2007) 8897. DOI:10.1073/pnas.0609568104

5. K. Kummerer, Antibiotics in the aquatic environment - A review - Part II, Chemosphere 75 (2009) 435. DOI:10.1016/j.chemosphere.2008.12.006

6. T. Deblonde, C. Cossu-Leguilleb, P. Hartemanna, Emerging pollutants in wastewater: A review of the literature, Int. J. Hyg. Envir. Heal. 214 (2011) 442. DOI:10.1016/j.ijheh.2011.08.002

7. S. A. Ozkan, Electroanalytical Methods in Pharmaceutical Analysis and their Validation, HNB Publishing, USA, 2011. ISBN:978-0-9664286-7-4 
8. T. Heberer, Occurrence, fate, and removal of pharmaceutical residues in the aquatic environment: a review of recent research data, Toxicol. Lett. 131 (2002) 5. DOI:10.1016/S0378-4274(02)00041-3

9. D. Nematollahi, H. Shayani-Jam, M. Alimoradi, S. Niroomand, Electrochemical oxidation of acetaminophen in aqueous solutions: Kinetic evaluation of hydrolysis, hydroxylation and dimerization processes, Electrochim. Acta 54 (2009) 7407. DOI:10.1016/j.electacta.2009.07.077

10. R. N. Goyal, S. P. Singh, Voltammetric determination of paracetamol at C60modified glassy carbon electrode, Electrochim. Acta 51 (2006) 3008. DOI:10.1016/j.electacta.2005.08.036

11. M-P. N. Bui, C. Ai Li, K. N. Han, X-H. Pham, G. H. Seong, Determination of acetaminophen by electrochemical co-deposition of glutamic acid and gold nanoparticles, Sens. Actuators, B 174 (2012) 318. DOI:10.1016/j.snb.2012.08.012

12. B. C. Lourencao, R. A. Medeiros, R. C. Rocha-Filho, L. H. Mazo, O. Fatibello-Filho, Simultaneous voltammetric determination of paracetamol and caffeine in pharmaceutical formulations using a boron-doped diamond electrode, Talanta, 78 (2009) 748. DOI:10.1016/j.talanta.2008.12.040

13. N. F. Atta, A. Galal, F. M. Abu-Attia, S. M. Azab, Simultaneous determination of paracetamol and neurotransmitters in biological fluids using a carbon paste sensor modified with gold nanoparticles, J. Mater. Chem. 21 (2011) 13015. DOI:10.1039/C1JM11795E 
14. C. Engin, S. Yilmaz, G. Saglikoglu, S. Yagmur, M. Sadikoglu, Electroanalytical Investigation of Paracetamol on Glassy Carbon Electrode by Voltammetry, Int. J. Electrochem. Sci., 10 (2015) 1916 - 1925.

15. Q. Zhao, Z. Zhang, L. Bao, D. Pang, Surface structure-related electrochemical behaviors of glassy carbon electrodes, Electrochem. Commun. 10 (2008) 181. DOI: 10.1016/j.elecom.2007.11.017

16. R. McCreery, K. Cline "10. Carbon Electrodes" pp. 293 in P.Kissinger, W. Heineman "Laboratory Techniques in Electroanalitycal Chemistry" II Ed. (1996) Marcel Dekker, Inc. New York ISBN 0-8247-9445-1

17. Dekanski, J. Stevanović, R. Stevanović, B. Ž. Nikolić, V. M. Jovanović, Glassy carbon electrodes: I. Characterization and electrochemical activation, Carbon 39 (2001) 1195. DOI:10.1016/S0008-6223(00)00228-1

18. X. Zhang, D. Zhang, Y. Chen, X. Sun, Y. Ma, Electrochemical reduction of graphene oxide films: Preparation, characterization and their electrochemical properties, Chin. Sci. Bull. 57 (2012) 3045. DOI:10.1007/s11434-012-5256-2

19. Joon I. Jang, New Developments in Photon and Materials Research: I. Childres, Chapter 19. Raman Spectroscopy of Graphene and Related Materials, NOVA Science Publisher, USA, 2013. ISBN: 978-1-62618-384-1

20. Vlad, N. Singh, J. Rolland, S. Melinte, P. M. Ajayan, J.F. Gohy, Hybrid supercapacitor-battery materials for fast electrochemical charge storage, Sci Rep. 4 (2014) 4315. DOI: 10.1038/srep04315 
21. H. Lindstrom, S. Sodergren, A. Solbrand, H. Rensmo, J. Hjelm, A. Hagfeldt, S. Lindquist Li+ Ion Insertion in TiO2 (Anatase). 2. Voltammetry on Nanoporous Films J. Phys. Chem. B 101 (1997), 7717

22. M. Zidan, T. W. Tee , A. H. Abdullah, Z. Zainal, G. J. Kheng Electrochemical Oxidation of Paracetamol Mediated by Nanoparticles Bismuth Oxide Modified Glassy Carbon Electrode, Int. J. Electrochem. Sci., 6 (2011) 279.

23. A. Ribeiro, M. Barros, L. Veríssimo, C. Santos, A. Cabral, G. Gaspar, M. Esteso Diffusion coefficients of paracetamol in aqueous solutions, J. Chem. Thermodynamics 54 (2012) 97. DOI: 10.1016/j.jct.2012.03.014

24. O. Laoire, E. Plichta, M. Hendrickson, S. Mukerjee, K.M. Abraham Electrochemical studies of ferrocene in a lithium ion conducting organic carbonate electrolyte, Electrochimica Acta 54 (2009) 6560. DOI: 10.1016/j.electacta.2009.06.041

25. Shi, F. C. Anson Multiple Intramolecular Electron Transfer in the Catalysis of the Reduction of Dioxygen by Cobalt meso-Tetrakis(4-pyridy1)porphyrin to Which Four $\mathrm{Ru}(\mathrm{NH} 3)$ Groups Are Coordinated, J. Am. Chem. Soc.113 (1991) 9564. DOI: $10.1021 / \mathrm{ja} 00025 \mathrm{a} 022$

26. K. Gadamsetti S. Swavey Electrocatalytic reduction of oxygen at electrodes coated with a bimetallic cobalt(II)/platinum(II) porphyrin, Dalton Trans., (2006) 5530. DOI: $10.1039 / \mathrm{b} 614108 \mathrm{k}$

27. X. ShangGuan, H. Zhang, J. Zheng Electrochemical behavior and differential pulse voltammetric determination of paracetamol at a carbon ionic liquid electrode, Anal Bioanal Chem 391 (2008) 1049. DOI: 10.1007/s00216-008-2096-7 
28. C. Van der Berg Determination of Copper, Cadmium and Lead in seawater by cathodic stripping voltammetry of complexes with 8-hydroxyquinoline, J. Electroanal. Chem. 215 (1986) 111. DOI:10.1016/0022-0728(86)87009-7 
Tables

Table 1 XPS atomic abundance from survey scan

\begin{tabular}{lll}
\hline & Atomic Abundance (\%) \\
& Virgin surface & Activated surface \\
\hline $\mathrm{C}$ & 92.3 & 66.5 \\
$\mathrm{O}$ & 6.7 & 30.5 \\
$\mathrm{~N}$ & 0.7 & 0.5 \\
$\mathrm{P}$ & 0.2 & 0.7 \\
$\mathrm{~S}$ & 0.1 & - \\
$\mathrm{B}$ & - & 1 \\
$\mathrm{Na}$ & - & 0.4 \\
$\mathrm{~F}$ & - & 0.3 \\
$\mathrm{O} / \mathrm{C}$ & 0.1 & 0.5 \\
\hline
\end{tabular}


Figures
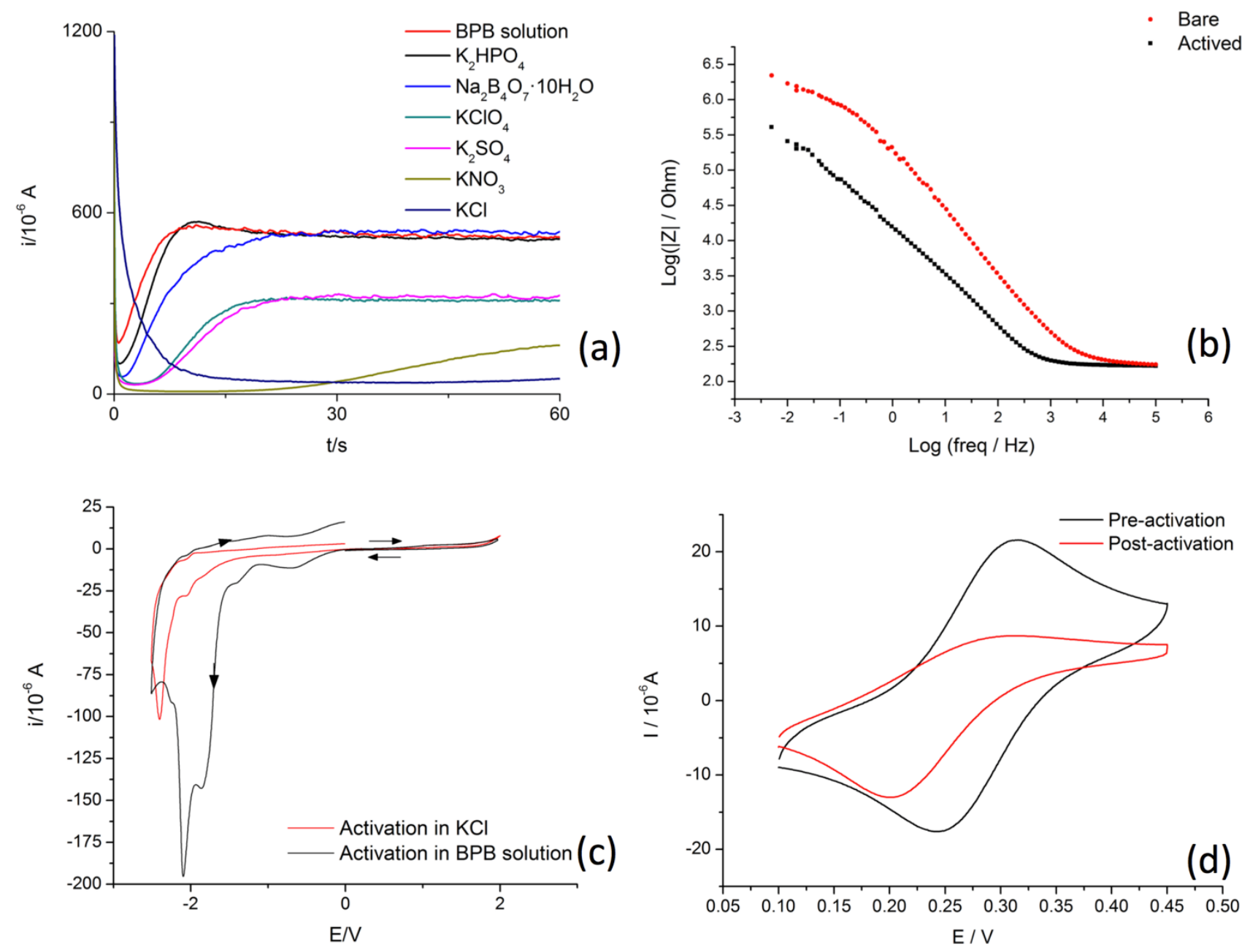

Figure 1 (a) Chronoamperometric curves of the GCE in different electrolytes $\left(5 \cdot 10^{-2} \mathrm{M}, \mathrm{pH}\right.$ 9); (b) Bode plots obtained prior and after the activation $\left(1 \cdot 10^{5}\right.$ to $2 \cdot 10^{-2} \mathrm{~Hz}$, sinusoidal amplitude $7 \cdot 10^{-3} \mathrm{~V}$ RMS) for $2 \cdot 10^{-5} \mathrm{M}$ AP in $\mathrm{BPB} 5 \cdot 10^{-2} \mathrm{M}$, pH 9; (c) $\mathrm{CV}$ of blank acetonitrile after the activation of $\mathrm{GCE}$ in $\mathrm{KCl} 50 \mathrm{mM}, \mathrm{pH} 9$ or BPB $50 \mathrm{mM}, \mathrm{pH} 9\left(\mathrm{CH}_{3} \mathrm{CN}\right.$,

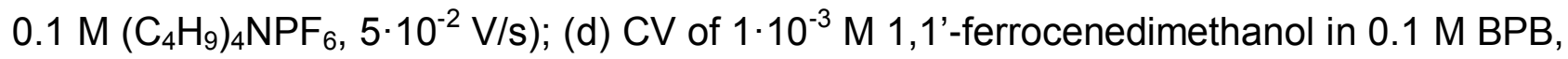
$\mathrm{pH}$ 9, before and after activation of the electrode. 

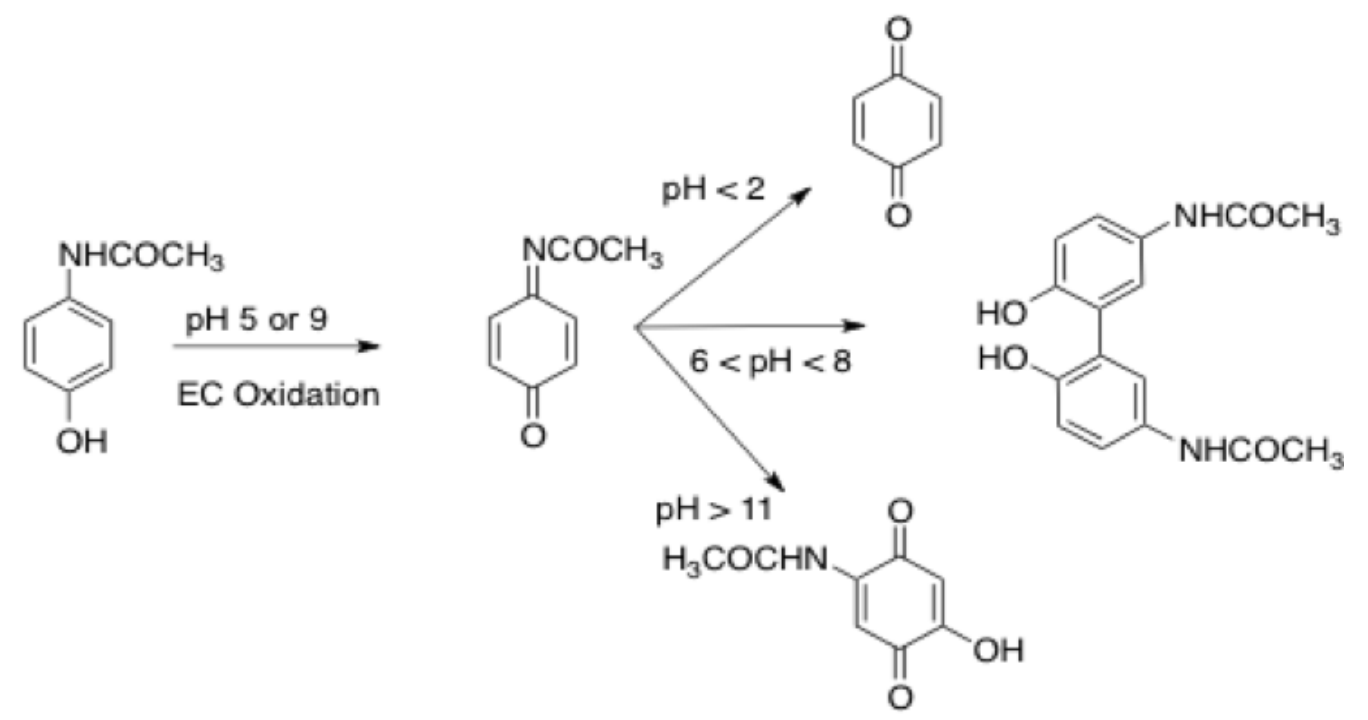

Figure 2 Scheme depicting the electrochemical reactivity of AP, as devised by [9].

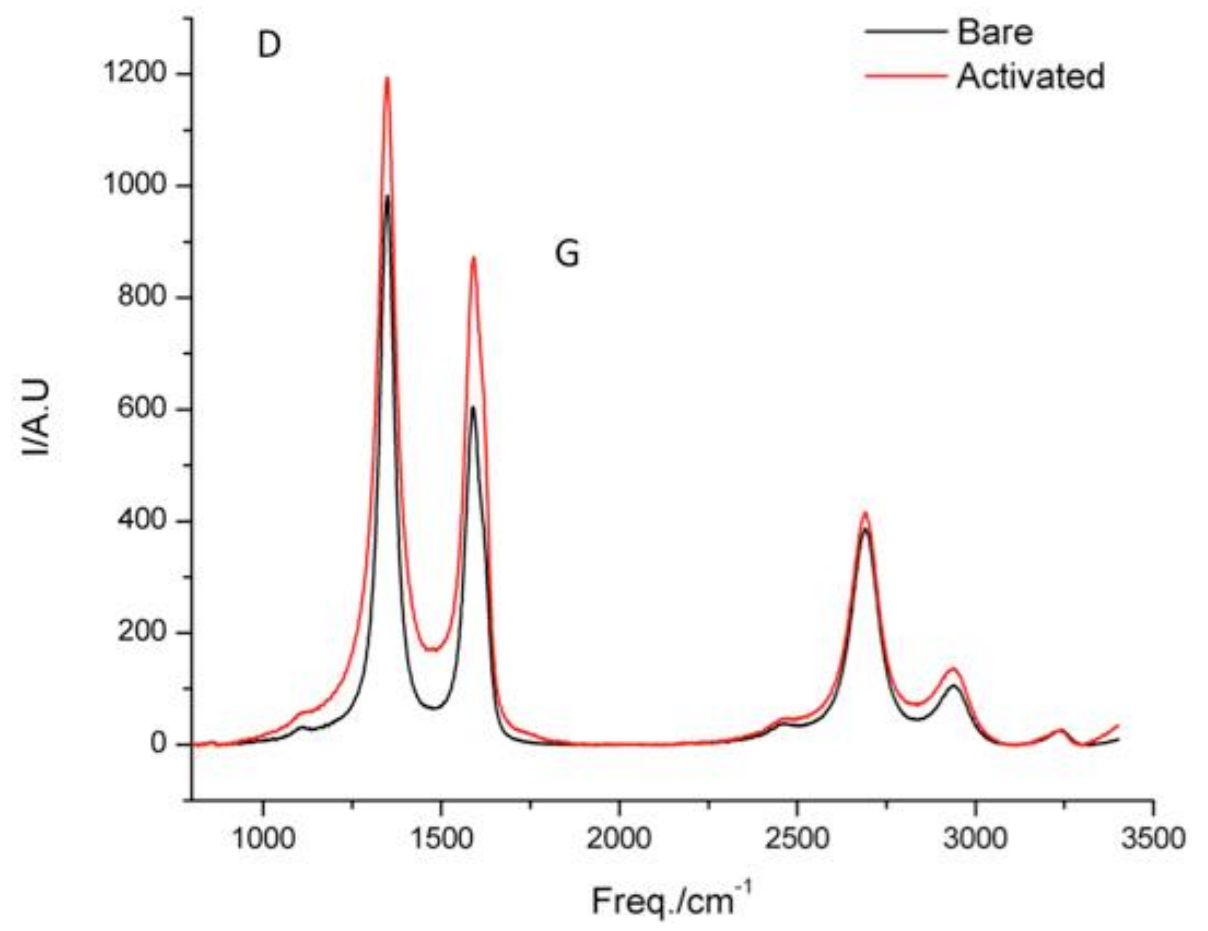

Figure 3 Raman spectra of the GC plate before (bare) and after the activation procedure $\left(\lambda_{\text {exc }} 532 \mathrm{~nm}, 100 \mathrm{X}\right)$; 

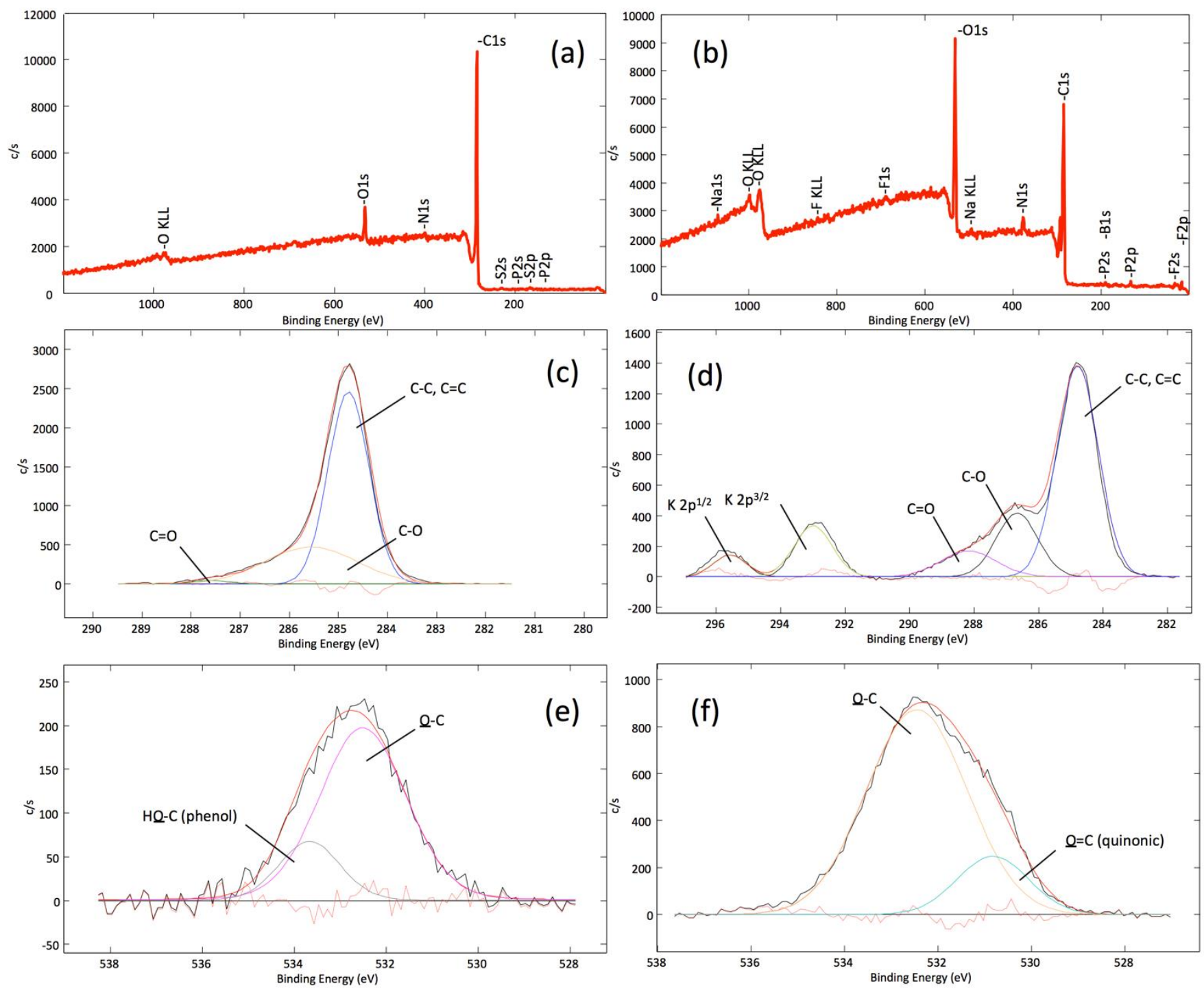

Figure 4 XPS spectrum in survey mode $(a, b)$ and in high resolution mode $(c, d, e, f)$ : in the middle the $\mathrm{C}_{1 \mathrm{~s}}$ region prior (c) and after (d) the activation; lower in the figure, the $\mathrm{O}_{1 \mathrm{~s}}$ region is shown prior (e) and after the activation (f); the deconvoluted curves are superimposed to the raw peak, showing the functional groups on the electrode surface. 

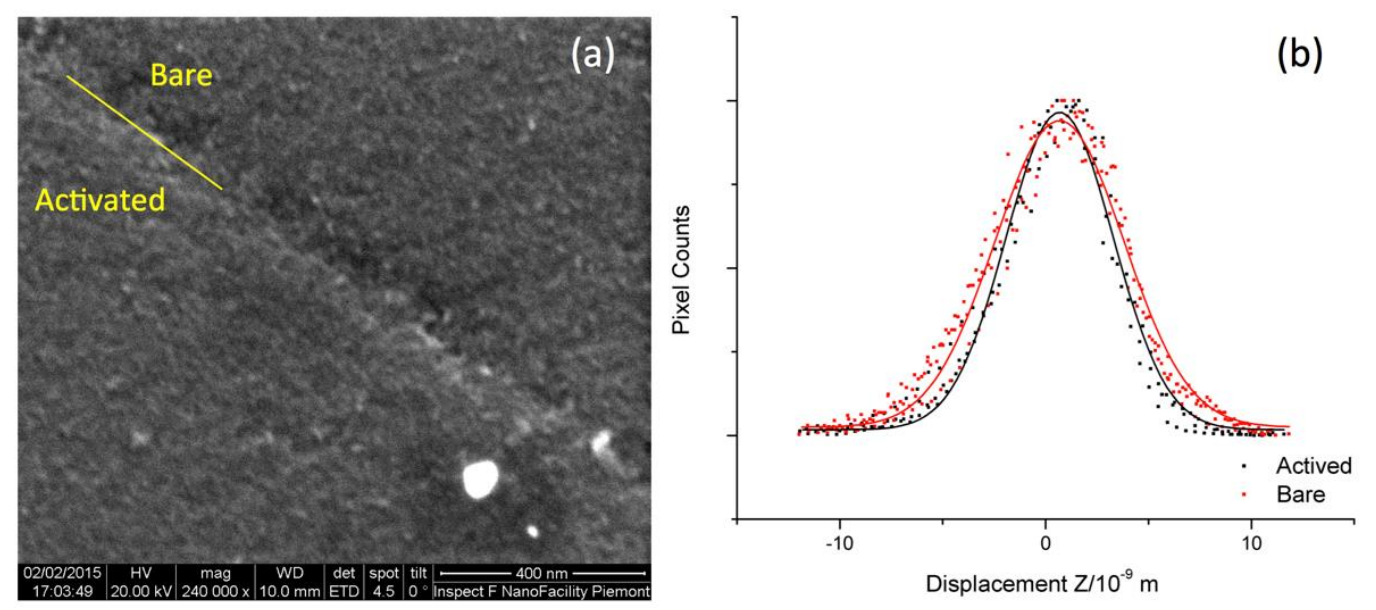

Figure 5 (a) SEM image of the GC plate: upper part, bare; lower part, activated (20 kV, 240000X); (b) Distribution of the Z-axis displacement of the surface derived from AFM measurements, mediated on different regions of the GC plate (bare and activated). 

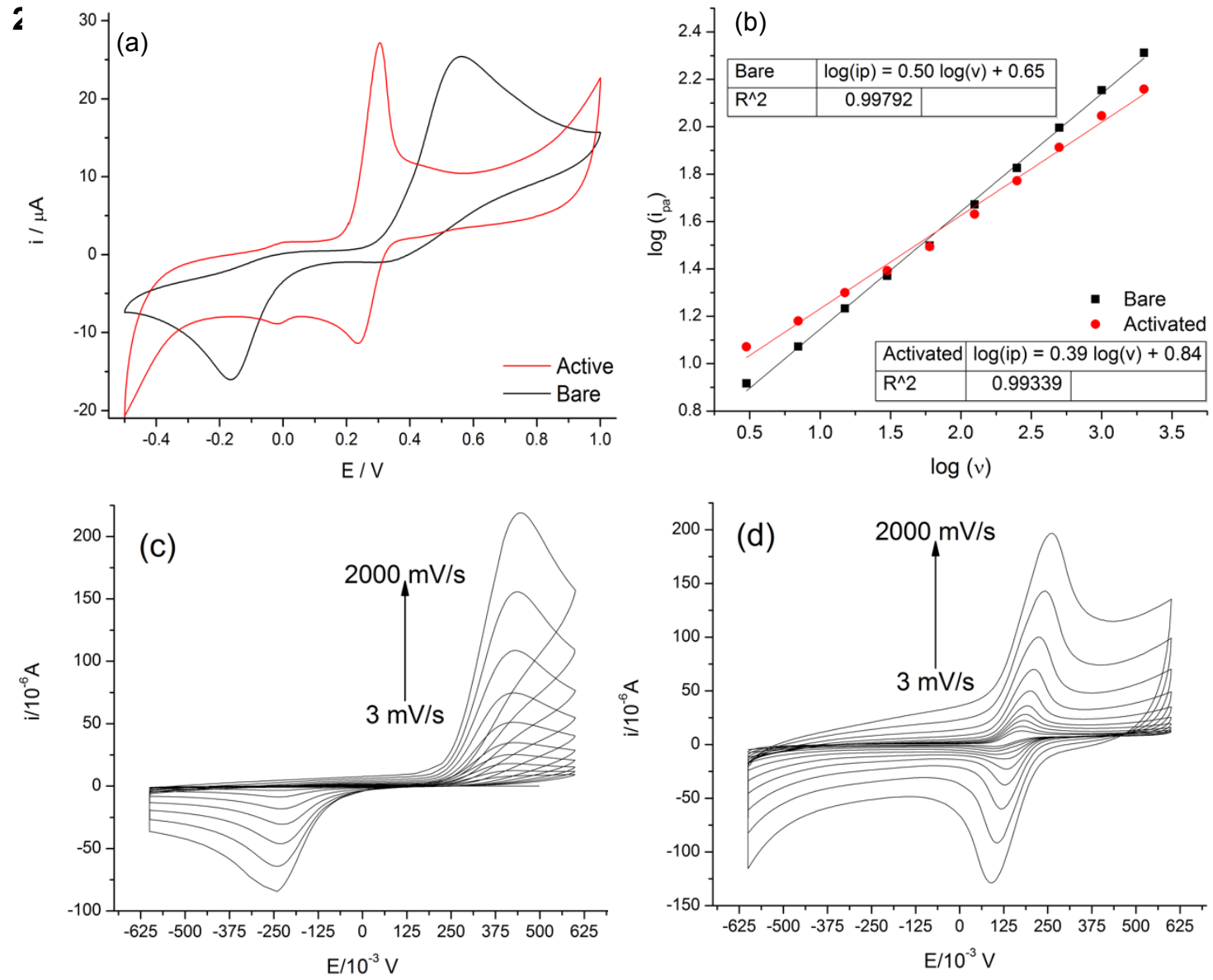

Figure 6 (a) CVs of AP $\left(1 \cdot 10^{-1} \mathrm{~V} / \mathrm{s}, \mathrm{AP} 1 \cdot 10^{-3} \mathrm{M}, 5 \cdot 10^{-2} \mathrm{M} \mathrm{BPB}, \mathrm{pH} 9\right)$, before and after activation of the electrode; (b) plot reporting $\log \left(i_{\mathrm{pa}}\right)$ vs $\log (v),(\mathrm{E}$ vs pseudo-Ag/AgCl, AP 2 $\cdot 10^{-3} \mathrm{M}, 5 \cdot 10^{-2} \mathrm{M}$ BPB, $\left.\mathrm{pH} 9\right) ; \mathrm{CVs}$ of $\mathrm{AP}$ at variable scan rate, prior (c) and after activation (d); 

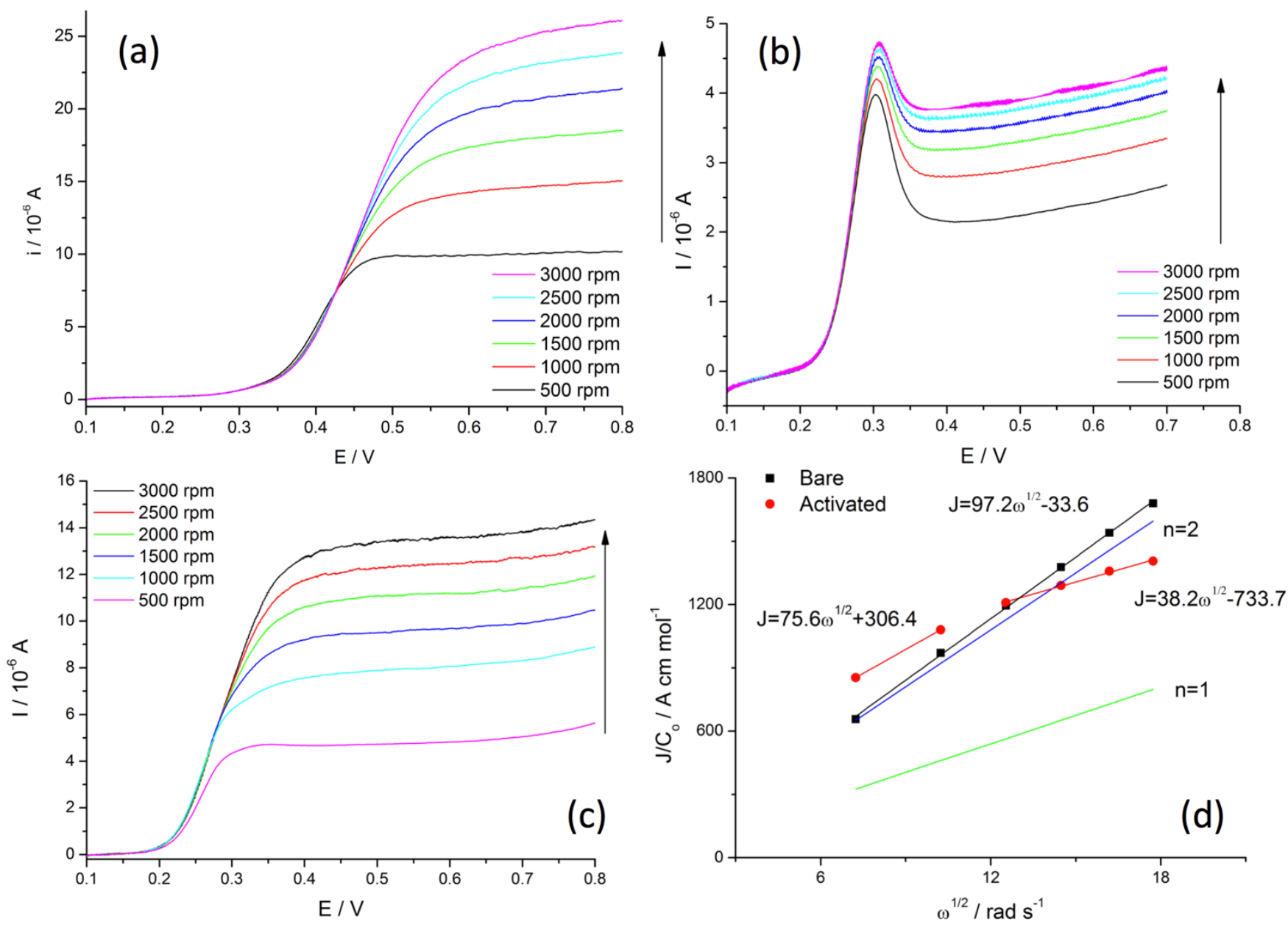

Figure 7 Levich study with RDE of $\mathrm{AP}\left(5 \cdot 10^{-2} \mathrm{~V} / \mathrm{s}, \mathrm{E}\right.$ vs $\mathrm{Ag} / \mathrm{AgCl}, \mathrm{KCl} 3 \mathrm{M}, \mathrm{AP} 2 \cdot 10^{-3} \mathrm{M}$, $5 \cdot 10^{-2} \mathrm{M} \mathrm{BPB}, \mathrm{pH} 9$ ): prior to activation (a), after activation (b) and after exposure to CTAB (c), by immersion in $5 \cdot 10^{-4} \mathrm{M} \mathrm{CTAB}$ solution for $1 \mathrm{~min}$. (d) Levich plot of molar current density. The symbols represent the experimental points together with the linear fits, while the continuos lines are predictions based on the Levich equation for the $\mathrm{n}=1$ (green) and $\mathrm{n}=2$ (blue) cases. 

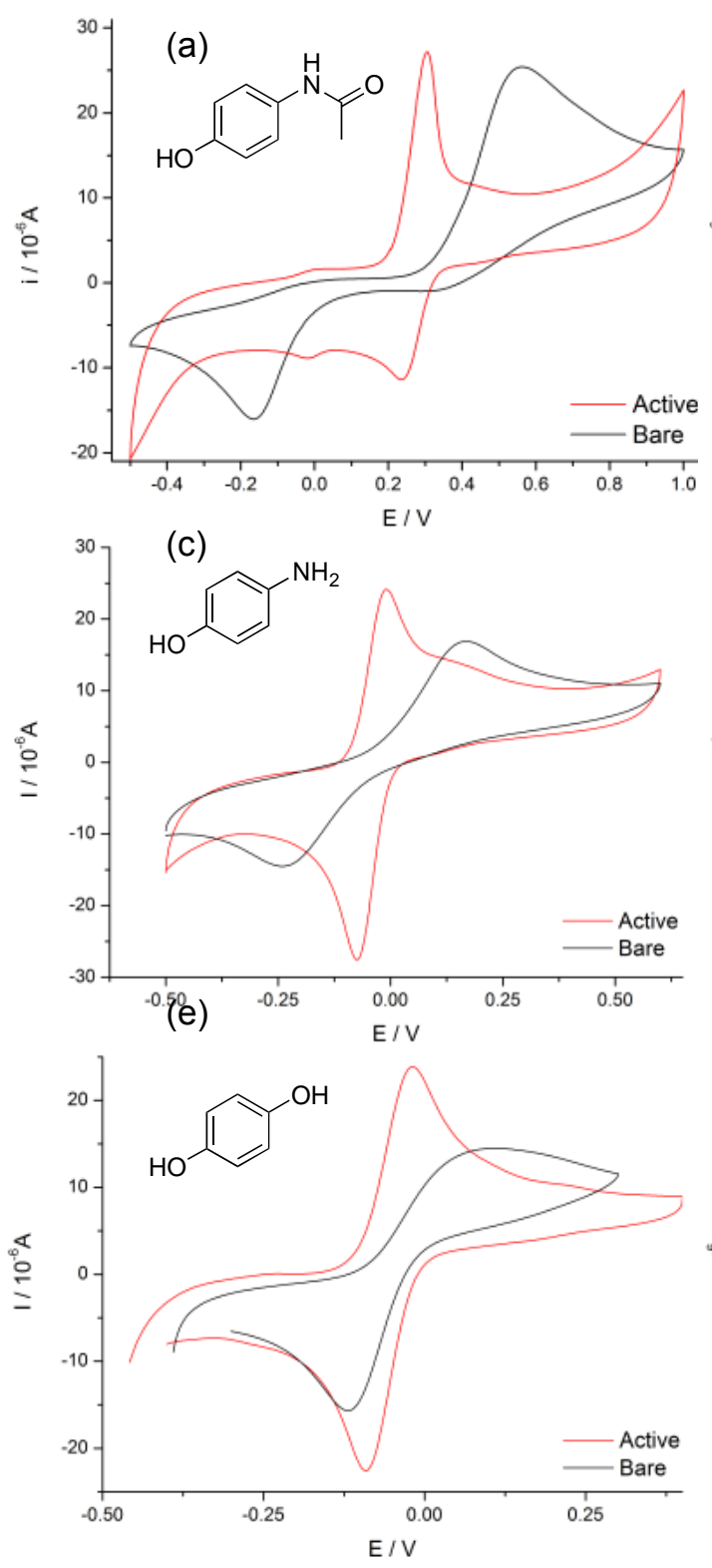

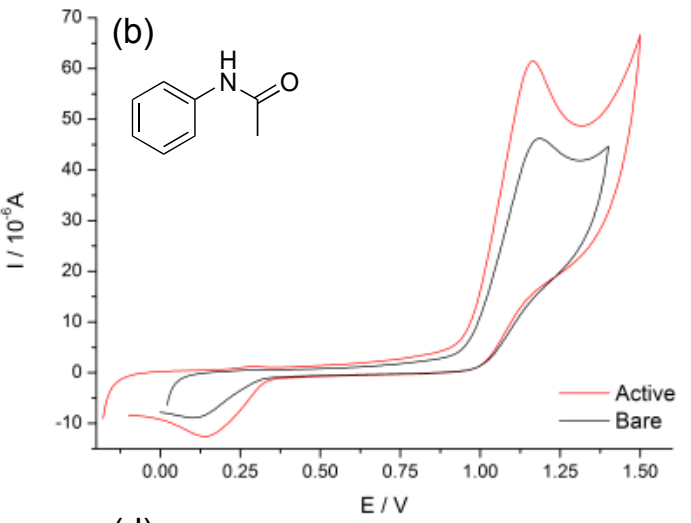

(d)
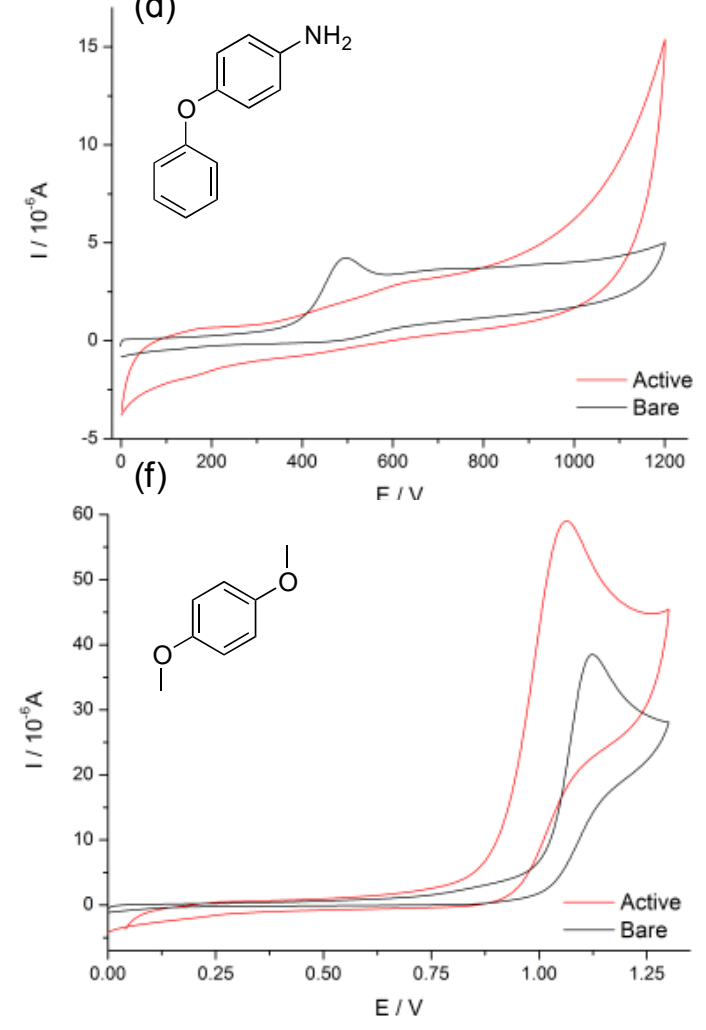

Figure $8 \mathrm{CV}$ curves of AP-mimiking molecule: (a) AP $\left(1 \cdot 10^{-3} \mathrm{M}\right)$, (b) acetanilide $\left(1 \cdot 10^{-3} \mathrm{M}\right)$, (c) p-aminophenol $\left(1 \cdot 10^{-4} \mathrm{M}\right),(\mathrm{d}) \mathrm{p}$-phenoxyaniline $\left(1 \cdot 10^{-4} \mathrm{M}\right)$, (e) hydroxyquinone $\left(1 \cdot 10^{-3}\right.$ $\mathrm{M})$, (f) hydroxyquinone dimethyl ether $\left(1 \cdot 10^{-3} \mathrm{M}\right)$. All the measurements has been performed in $5 \cdot 10^{-2} \mathrm{M} \mathrm{BPB}, \mathrm{pH} 9,1 \cdot 10^{-1} \mathrm{~V} / \mathrm{s}$. 

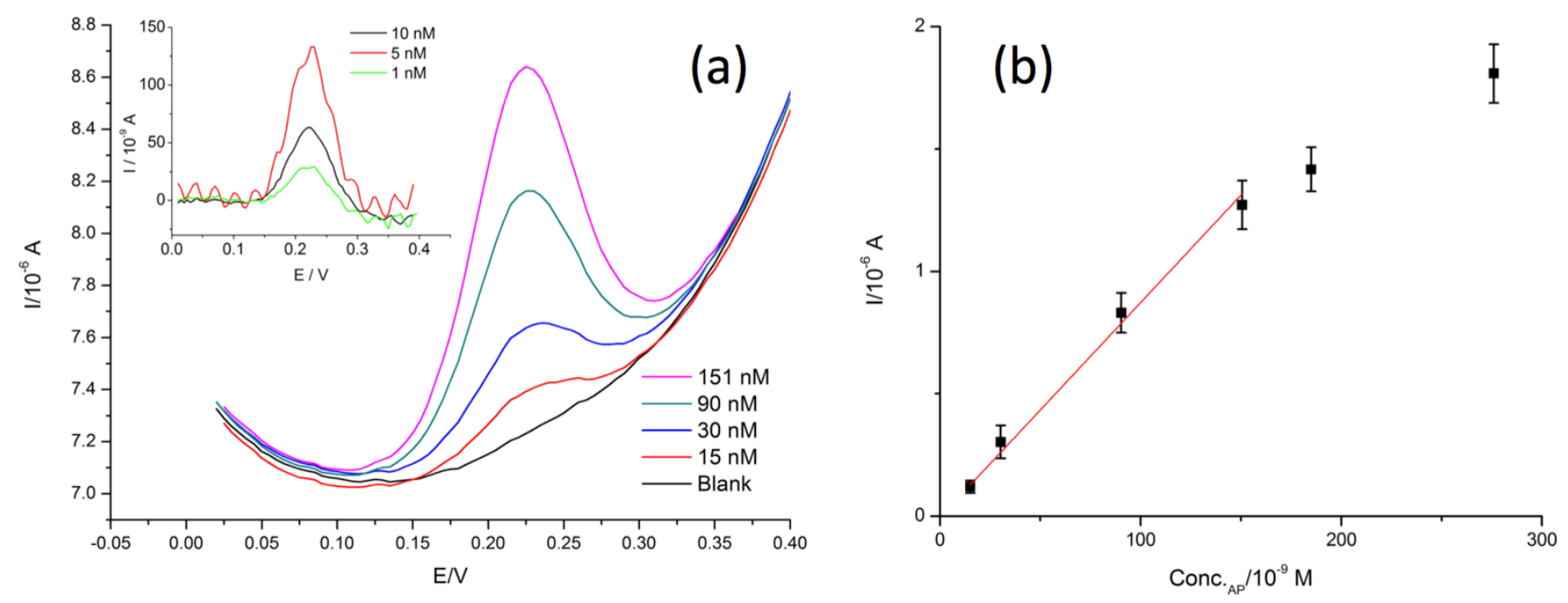

Figure 9 (a) DPV curves for the full range of AP concentrations $\left(0-1.5 \cdot 10^{-7} \mathrm{M}\right)$ and (inset) DPV curves for low concentrations of AP $\left(1 \cdot 10^{-9}-1 \cdot 10^{-8} \mathrm{M}\right)$; (b) peak area, A, vs AP concentration (AP $0-2.9 \cdot 10^{-7} \mathrm{M}$ ) and calibration curve (external standard) calculated in the linearity range (AP $\left.0-1.5 \cdot 10^{-7} \mathrm{M}\right)$. Activation in $5 \cdot 10^{-2} \mathrm{M} \mathrm{BPB}, \mathrm{pH} 9$. 